\title{
Antoine Robitaille
}

politologue, journaliste, Le Devoir, Montréal

\author{
(9 décembre 2006)
}

\section{"Dion contre Dion. Léon Dion serait-il déçu en voyant son fils bloquer ce qu'il a cherché à fonder?"}

\footnotetext{
Un document produit en version numérique par Jean-Marie Tremblay, bénévole, professeur de sociologie au Cégep de Chicoutimi

Courriel: jean-marie tremblay@uqac.ca

Site web pédagogique : http://www.uqac.ca/jmt-sociologue/
}

Dans le cadre de la collection: "Les classiques des sciences sociales"

Site web: http://classiques.uqac.ca/

Une collection développée en collaboration avec la Bibliothèque

Paul-Émile-Boulet de l'Université du Québec à Chicoutimi

Site web: http://bibliotheque.uqac.ca/ 
Cette édition électronique a été réalisée par Jean-Marie Tremblay, bénévole, professeur de sociologie au Cégep de Chicoutimi à partir de :

Antoine Robitaille,

"Dion contre Dion. Léon Dion serait-il déçu en voyant son fils bloquer ce qu'il a cherché à fonder?"

Le Devoir, Montréal, édition du samedi 9 décembre 2006, pages A1 et A4A5.

L'auteur est journaliste au journal LE DEVOIR.

[L'auteur nous a permis, samedi, le 9 décembre 2006, de diffuser le texte intégral de cet article dans Les Classiques des sciences sociales.]

81 Courriels : arobitaille1@,videotron.ca ou arobitaille@,1edevoir.com

Polices de caractères utilisée :

Pour le texte: Times New Roman, 14 points.

Pour les citations : Times New Roman, 12 points.

Pour les notes de bas de page : Times New Roman, 12 points.

Édition électronique réalisée avec le traitement de textes Microsoft Word 2004 pour Macintosh.

Mise en page sur papier format : LETTRE (US letter), 8.5' x 11',)

Édition numérique réalisée le 11 décembre 2006 à Chicoutimi, Ville de Saguenay, province de Québec, Canada.

\section{CFait avec}


Voir les œuvres de politologues québécois disponibles dans Les Classiques des sciences sociales :

Louis Balthazar, Université Laval; Gérard Bergeron, Université Laval; Roch Denis, UQÀM; Léon Dion, Université Laval; Alain-G. Gagnon, UQÀM; Diane Lamoureux, Université Laval; Vincent Lemieux, Université Laval; Denis Monière, Université de Montréal; Jean-Marc Piotte. UQÀM;

Jean-Marie Tremblay, sociologue Fondateur, Les Classiques des sciences sociales. 
Antoine Robitaille,

“Dion contre Dion. Léon Dion serait-il déçu en voyant son fils bloquer ce qu'il a cherché à fonder?".

Le Devoir, Montréal, édition du samedi 9 décembre 2006, pages A1 et A4-A5.

\section{Introduction}

Texte sous la photo : Léon et Stéphane Dion: deux politologues. L'un, le père, profondément hésitant face à la politique et au dilemme QuébecCanada. L'autre, le fils, qui plonge, devient un requin de la politique et un faucon de l'unité canadienne.

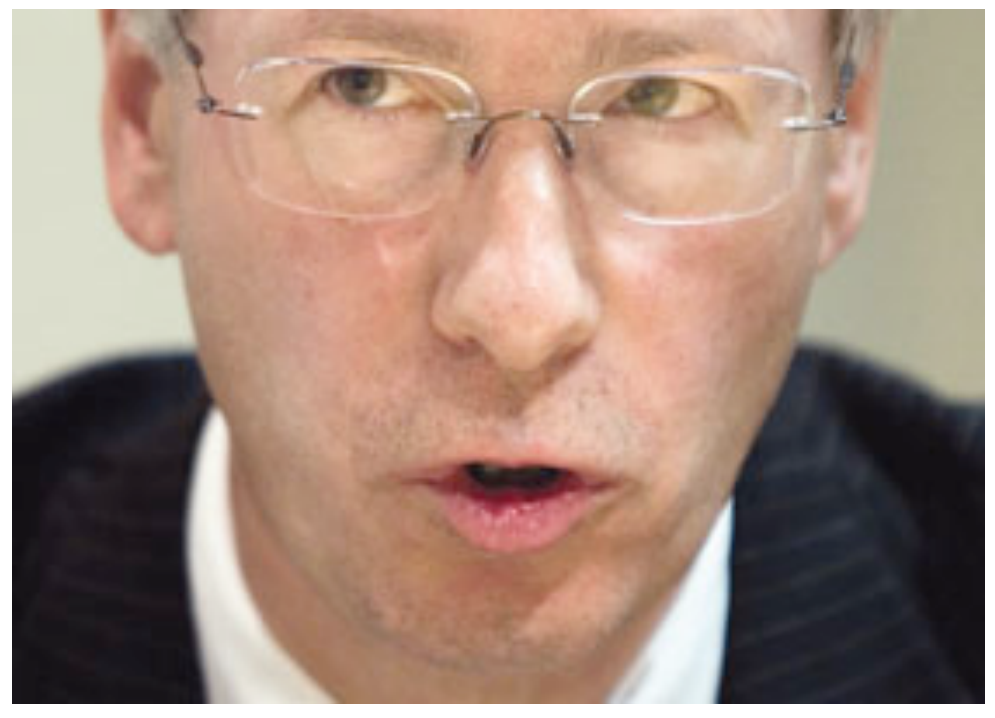
Comment peut-on interpréter leur rapport sur le plan intellectuel? Que cela nous dit-il des défis qui attendent le nouveau chef du PLC?

Québec -- «Tuer le père»: telle est une des métaphores les plus rebattues de la littérature contemporaine. C'est par un angle apparenté que plusieurs interprètent le personnage de Stéphane Dion, surtout dans les cercles souverainistes, exaspérés tant par le «fédéraliste fatigué» que par le «fédéraliste fatigant» -- comme on les y appelle. Une légende inspirée de ce thème freudien s'y est même forgée: Léon Dion, célèbre politologue de l'Université Laval, serait mort chagriné à la vue de son fils qui bloquait en politique ce qu'il avait cherché à fonder intellectuellement. 
Dans la famille Dion, on rejette évidemment ce type d'interprétation toute caricaturale. "Tout ce que les gens peuvent dire... c'est incroyable», laisse tomber Denyse Dion à l'autre bout du fil, légèrement découragée, mais tout de même en verve. Le jour précédent, la femme de 77 ans avait pris position fermement dans la polémique sur la citoyenneté française de son fils: "Qu'il y renonce s'il le veut. Je m'en moque absolument. Je la trouve tellement idiote, cette histoire.» Sur les rapports entre le père et le fils, cette Parisienne d'origine qui a élevé quatre garçons et une fille se montre aussi catégorique: «De tous mes enfants, aucun n'a peut-être été aussi près de mon mari. Intellectuellement, s'entend. Adolescent, Stéphane discutait constamment avec son père.»

Le frère aîné de Stéphane, Patrice Dion, professeur de phytologie à l'Université Laval, se désole aussi de cette «psychologie facile» qui consiste à opposer le politologue père au fils politologue-politicien. «Ils avaient une très belle relation. Stéphane consultait beaucoup mon père. Même s'ils ne s'entendaient pas sur tout, il n'y avait aucune espèce de conflit entre les deux.» En année sabbatique à l'étranger lorsque Stéphane entre aux affaires en 1996, Patrice Dion se souvient des nouvelles qu'il avait eues à l'époque: son père «était extrêmement heureux» à l'idée de voir Stéphane plonger. «Mon frère et ma soeur m'avaient même dit à l'époque qu'il "vivait une nouvelle jeunesse".»

Reste que, sur cette question, difficile de savoir quelle a vraiment été la position de Léon Dion -- comme souvent, diront les mauvaises langues -- puisque les versions divergent. Denyse Dion, entre autres, hésite. Globalement, le père était plutôt soucieux de voir le fils s'embarquer dans un «monde difficile», raconte-t-elle; mais il en était très fier. «Moi, en tout cas, j'étais déçue!», tranche-t-elle encore. Son fils, à ses yeux, avait «une carrière formidable», «était demandé dans tous les pays». Il laissait tomber tout cela pour la politique, un domaine pour lequel elle dit avoir développé une aversion pendant la Seconde Guerre mondiale. "Vous savez, c'était difficile, l'occupation», se souvient celle qui a grandi près des Buttes-Chaumont, dans le XIXe arrondissement. «J'ai souffert toute ma vie de la politique. De ces débats sans fin entre pétainistes, gaullistes, etc.» En politique, déplore-t-elle, il n'y a pas de place pour le gris. "Tout est soit blanc, soit noir», ditelle, avant d'ajouter que son fils «échappe sans doute à cela». 
Vincent Lemieux, collègue et ami de Léon Dion, a pour souvenir, lui, un Léon Dion plutôt déçu, «les premiers jours du moins», de voir son fils quitter la vie universitaire, lui qui avait plusieurs fois résisté à l'appel de la politique. «Il lui avait dit, avec son humour... beaucoup plus subtil que celui de Stéphane, en passant... que les politiciens allaient toujours voir en lui un universitaire et que les universitaires allaient toujours voir en lui un politicien.»

On raconte que Léon Dion n'a jamais fait de politique à cause de son épouse, qui refusait. «Oh, il se servait de moi comme paravent, vous savez», répond Denyse Dion. «S'il l'avait vraiment voulu, je l'aurais laissé y aller.» Stéphane et Léon sont deux personnes très différentes, souligne-t-elle. «Léon aimait recevoir des politiciens de toutes les tendances, se mettait à leur place et tentait de les comprendre. À un moment, il a fait une incursion de l'autre côté. Dans les années 80, il est devenu conseiller [du ministre libéral] Gil Rémillard. Ce fut les pires mois de sa vie. Je ne l'ai jamais vu aussi malheureux. Il n'aurait pas duré longtemps en politique parce qu'il fallait faire des compromis, dit-elle. Stéphane, lui, a un tempérament différent.» À sa façon, lui aussi n'est pas très porté sur les compromis, non? «Vous savez, la politique, ça vous moule, il est beaucoup plus souple qu'avant.»

\section{Un mémoire de maîtrise sur le $P Q$}

Vincent Lemieux, qui a dirigé le mémoire de maîtrise de Stéphane Dion à Laval dans les années 70, se souvient d'un étudiant assez effacé et peu politisé: «Ce n'est jamais facile pour un enfant d'un professeur d'étudier à l'endroit où enseigne papa», dit M. Lemieux. Patrice Dion rappelle aussi qu'à l'époque, à Laval, «c'était une période bizarre, en sciences sociales, pas mal marxiste-léniniste. Mon père trouvait cela difficile, se faisait tutoyer et apostropher par des barbus dans les auditoriums», illustre-t-il. C'est au mouvement souverainiste toutefois que vont les sympathies de l'étudiant Dion, se souvient Vincent Lemieux. «Un peu pour braver son père», il choisit donc de consacrer son mémoire de maîtrise au Parti québécois. 
En 1979, il dépose un texte de 132 pages: «La dimension temporelle de l'action partisane: l'étude d'un cas: le débat au sein du Parti québécois sur les modalités d'accession à l'indépendance». Eh oui, le chef du PLC est un «expert» des premières années du PQ! Dans son mémoire, il se penche entre autres sur «l'évolution du contenu de la publicité électorale» du PQ des campagnes de 1970, 1973 et 1976. Il décortique les débats des congrès de 1973, 1974 et de 1975 tout en analysant le réel avec des concepts politologiques très abstraits. À la page 7, il expose différentes théories sur les partis politiques, notamment celles de Vincent Lemieux et de... Léon Dion. Commentaire de l'étudiant: «Je propose de réarticuler à partir de la dimension temporelle cette façon classique de dichotomiser les partis politiques.» Autrement dit, le Stéphane Dion de 23 ans est déjà prêt à «corriger» le père et le professeur et à proposer sa propre grille!

\section{À l'UdeM}

«On supposait qu'il était de notre bord!», dit en rigolant le professeur à la retraite (et ancien président de la Société Saint-Jean Baptiste de Montréal) Guy Bouthiller à propos du moment, au milieu des années 80 , où Stéphane Dion a été embauché en science politique à l'Université de Montréal. (Chez les Dion d'ailleurs, au moins un enfant, Patrice, confie qu'il a toujours voté PQ et Bloc. Léon avait d'ailleurs incité à voter OUI en $1980 \ldots$ pour obtenir un fédéralisme renouvelé.)

$\mathrm{Au}$ doctorat, Dion a travaillé sur les banlieues communistes en France avec Michel Crozier. En 1986, ce dernier avait préfacé un livre tiré de sa thèse et intitulé La Politisation des mairies (Economica, 1986). (La thèse de Léon Dion portait, elle, sur l'autre extrémité du spectre idéologique, le national-socialisme de Hitler.)

Tous les midis ou presque pendant 12 ans, un petit groupe de professeurs, autour du trio Stéphane Dion, Denis Monière et André Blais, déjeunent ensemble à la cafétéria. Denis Monière se rappelle les discussions animées. "C'était un excellent prof; dommage qu'il soit parti en politique», dit en souriant le très souverainiste Monière. Stéphane Dion a du succès auprès de ses étudiants et reste en contact avec plu- 
sieurs d'entre eux. (Le très connu porte-parole de Greenpeace, Steven Guilbault, est un de ceux-là. Or, en 2005, au terme de la conférence de Montréal sur les changements climatiques, Guilbault a contribué à faire évoluer l'image de Dion en soulignant à plusieurs reprises sa maestria à titre de président de cette rencontre internationale.)

Concernant les rapports entre le fils et le père Dion, Denis Monière rejette évidemment la métaphore du «parricide». «N'allons pas dans les grands mots!» Toutefois, ajoute le professeur (expert d'un des amis marquants de Léon Dion, André Laurendeau), «les deux sont aux antipodes du fédéralisme». C'est au sortir de Meech, en 1990, que Stéphane Dion devient fédéraliste, contrairement à de nombreux Québécois. À l'époque, il revenait d'une année sabbatique à Washington, où il avait «enfin appris l'anglais», comme le raconte un ancien collègue, et où il avait couché sur papier sa thèse selon laquelle le Canada était la fédération la «plus décentralisée» du monde.

«Mon interprétation, dit Denis Monière à propos de la position fédéraliste de Dion fils, c'est qu'il a réagi à une stratégie proposée par son père et adoptée alors par Robert Bourassa, celle du "couteau sous la gorge"», cette idée selon laquelle il faut menacer le Canada anglais avec la souveraineté pour qu'il fasse des concessions constitutionnelles au Québec. Au dire de Monière, Dion fils croit que les Québécois sont tellement ambivalents qu'ils n'auront jamais le cran de se séparer. Autrement dit, pourquoi le Canada leur ferait-il des concessions? Ce dernier doit opter pour le «pari de la franchise» afin d'obliger les nationalistes mous à cesser d'osciller entre autonomie et adhésion pleine à la fédération. «En somme, il veut empêcher les Québécois de devenir des Léon Dion!», dit Monière. En cela, il appliquerait la théorie des jeux, une approche mathématique des problèmes de stratégie chère à un autre de ses professeurs de Laval, Jean-Pierre Derriennic. Celle-ci enseigne grosso modo que «celui qui gagne est celui qui prend la position la plus radicale», résume Monière en ajoutant, un brin cynique, «pour l'instant, ç'a fonctionné». 


\section{Nationalisme et libéralisme}

Un des auteurs qui ont le plus marqué Stéphane Dion est Alexis de Tocqueville. Entre autres parce qu'il lui a peut-être permis de sortir d'une des impasses dans lesquelles son père s'est empêtré: la tension entre le nationalisme et le libéralisme. Tension, en fait, entre le particulier et l'universel. C'est ce que suggère le philosophe Georges Leroux, dans un texte récent (revue Cité, $\mathrm{n}^{\circ} 23$ ). «Léon Dion est resté prisonnier de son tourment», soutient le politologue Guy Laforest, puisqu'il a été incapable de définir une réelle synthèse entre ces deux pôles. "Stéphane croit s'être affranchi de cette souffrance», entre autres en défendant les lois linguistiques (ici, comme son père, il défend fermement la protection linguistique particulière pour le Québec) dans un Canada uni, explique Laforest. Mais, selon ce dernier, lorsqu'on lit Dion fils, on trouve des «zones grises, des tensions non résolues, comme chez son père». Et certaines d'entre elles le placent «sur une ligne de collision avec le courant trudeauiste radical qui le soutient actuellement». Autrement dit, les tourments de Léon pourraient bien, tôt ou tard, rattraper Stéphane.

Fin du texte 